\title{
Seeded Growth of Nanoscale Semiconductor Tetrapods: Generality and the Role of Cation Exchange
}

Michael J. Enright ${ }^{\dagger}$, Florence Y. Dou, Shenwei Wu, Emily J. Rabe, Madison Monahan, Max R. Friedfeld, Cody W. Schlenker, Brandi M. Cossairt*

University of Washington, Department of Chemistry, Box 351700, Seattle, WA 98195-1700.

†Present Address: University of Illinois at Urbana-Champaign, School of Chemical Sciences, Urbana, IL 61801.
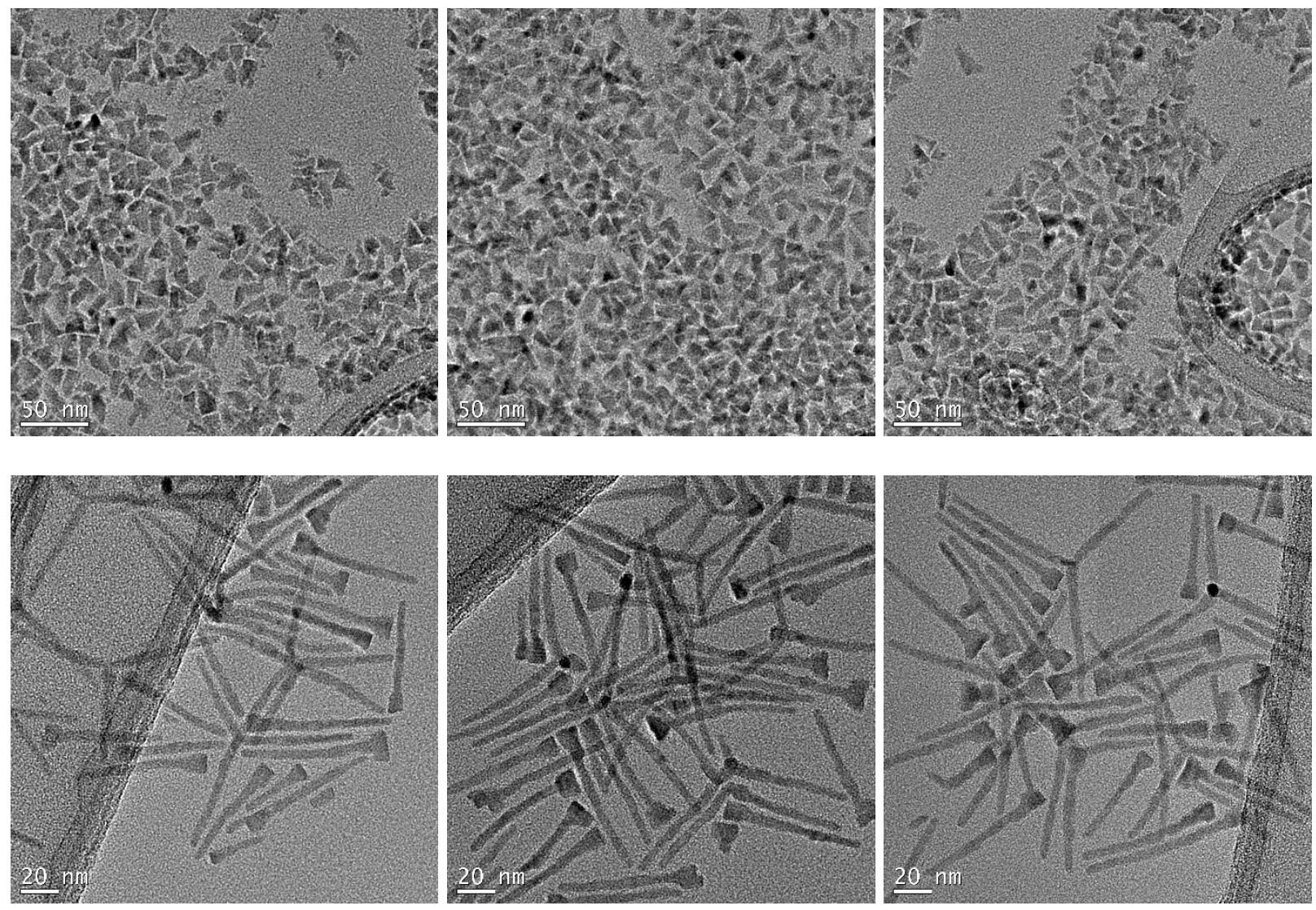

Figure S1. Example ensemble TEM images of CdS arm growth in absence of seed QD. Less than $1 \%$ (one tetrapod observed per 100 arms) tetrapods are found in the thick arm motif (top row) while less than $5 \%$ (about 7 tetrapods observed per 150 arms) are found in the thin arm motif (bottom row). 
Table S1. CdS arm dimensions for all tetrapods. For each sample, a minimum of 150 individual structures were evaluated. Accurate evaluation of length and width of nanoscale tetrapod samples is challenging from $2 \mathrm{D}$ projections. The accuracy of these measurements has inherent sources of unavoidable error. First, all tetrapod arms do not lie parallel to the imaging plane. Second, all tetrapods within a sample do not share the same orientation when deposited on TEM grids. These dimensions are measured and reported to the best of our ability from 2D projections of 3D samples. Arm length is measured from the edge of the core to the end of the arm. Arm width is measured across the thickest section of the arm, usually at the base of the arm on the edge of the core for thick tetrapod samples. Lengths and widths are reported in nanometers with standard deviations in ensemble sample measurements. While width measurements are relatively similar across samples, length measurements have higher variability. We attribute this variability to differences in seed surface and lattice parameters across core materials and sizes.

\begin{tabular}{ccccc}
\hline & \multicolumn{2}{c}{ Thin arms } & \multicolumn{2}{c}{ Thick arms } \\
Seed & Length (nm) & Width $(\mathrm{nm})$ & Length (nm) & Width (nm) \\
\hline CdS (unseeded arms) & $59 \pm 8$ & $4.5 \pm 0.6$ & $18 \pm 2$ & $10 \pm 2$ \\
ZnTe & $47 \pm 5$ & $5.9 \pm 0.7$ & $26 \pm 4$ & $9.1 \pm 1.2$ \\
CIS (3 nm) & $59 \pm 17$ & $5.5 \pm 0.7$ & $18 \pm 2$ & $9.2 \pm 1.2$ \\
CIS (6 nm) & $81 \pm 15$ & $4.6 \pm 0.6$ & $20 \pm 4$ & $9.6 \pm 1.8$ \\
InP (spherical) & $88 \pm 10$ & $5.5 \pm 0.6$ & $23 \pm 4$ & $10 \pm 2$ \\
InP (tetrahedral morphology) & $49 \pm 6$ & $4.7 \pm 0.6$ & $22 \pm 3$ & $8 \pm 1$ \\
InP/ZnSeS (69\% PLQY) & $16 \pm 2$ & $4.0 \pm 0.6$ & $12 \pm 1$ & $6.4 \pm 0.9$ \\
InP/ZnS (thick shell) & $12 \pm 1$ & $4.3 \pm 0.5$ & $10 \pm 1$ & $6.5 \pm 0.9$ \\
Cd $_{3} P_{2}$ & $42 \pm 5$ & $5.2 \pm 0.7$ & $15 \pm 2$ & $7.7 \pm 0.9$ \\
\hline
\end{tabular}




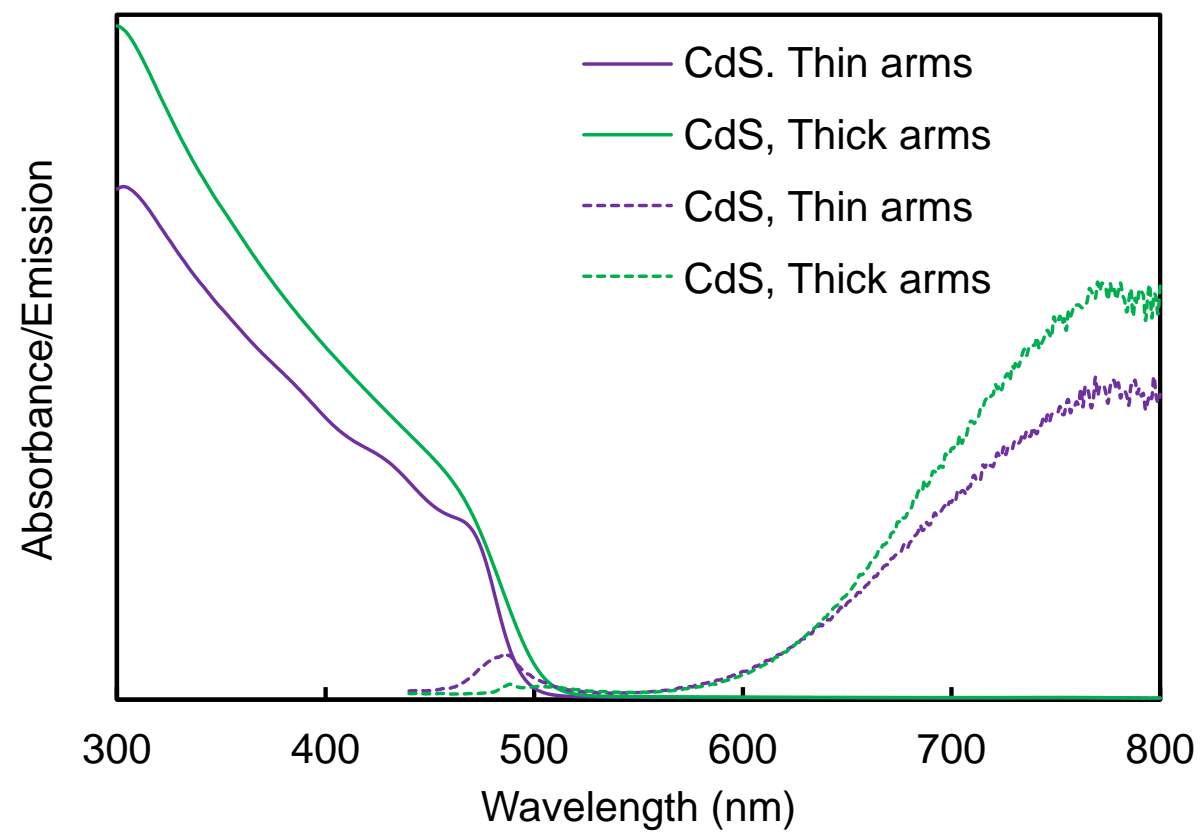

Figure S2. The absorbance (solid lines) and fluorescence (dotted lines) for CdS arm growth (no seed).
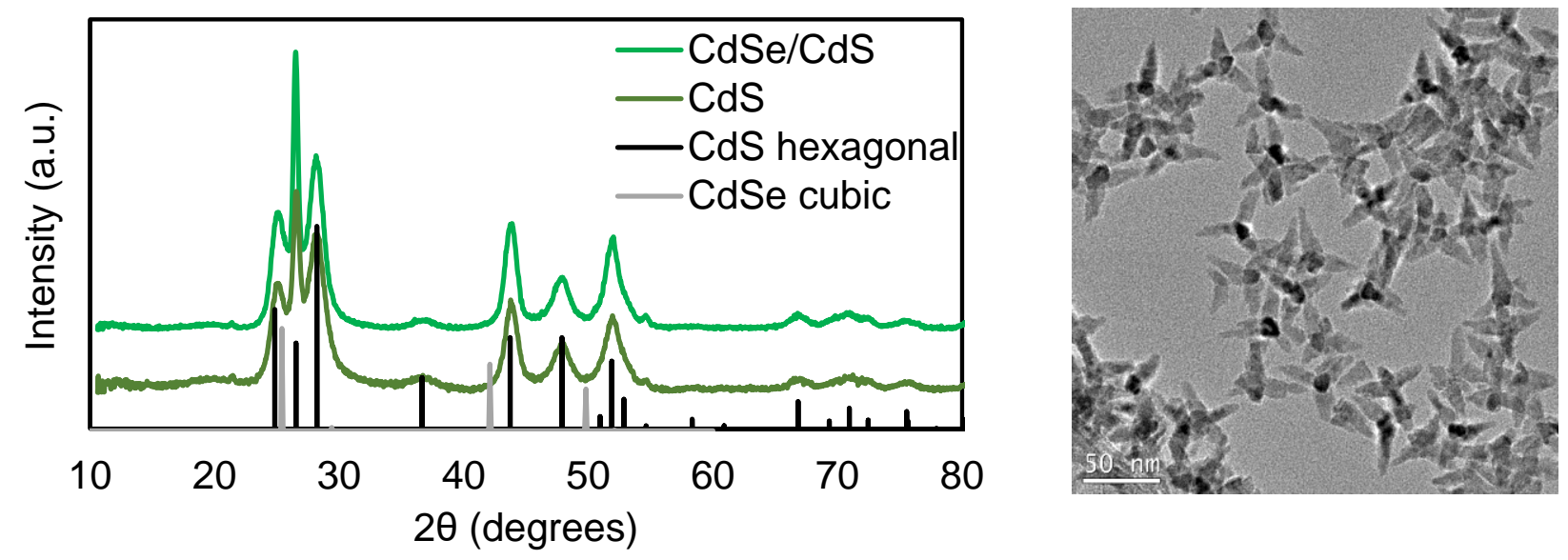

Figure S3. Growth of CdS arm growth upon CdSe seeds. XRD shows no signal from CdSe cores. Accompanying TEM of CdSe seeded $\mathrm{CdSe} / \mathrm{CdS}$ tetrapods shows independent $\mathrm{CdS}$ arms are not present after tetrapod synthesis. CdSe/CdS tetrapods are used as an example since the system is well studied, cannot undergo cation exchange, and retains a CdSe core. 


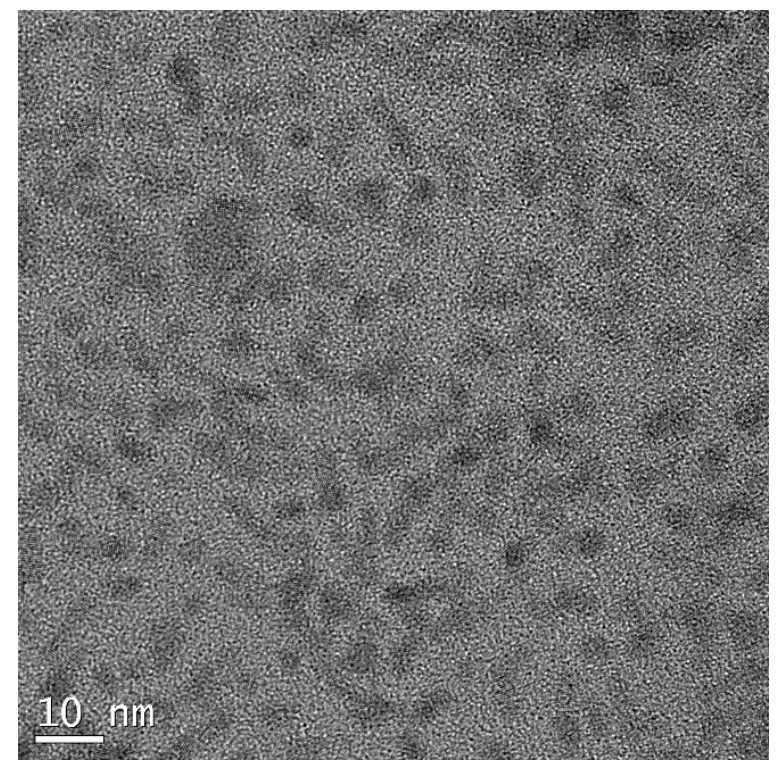

Figure S4. TEM of ZnTe Seeds. $8 \mathrm{~nm}$ with an aspect ratio of $\sim 2$.

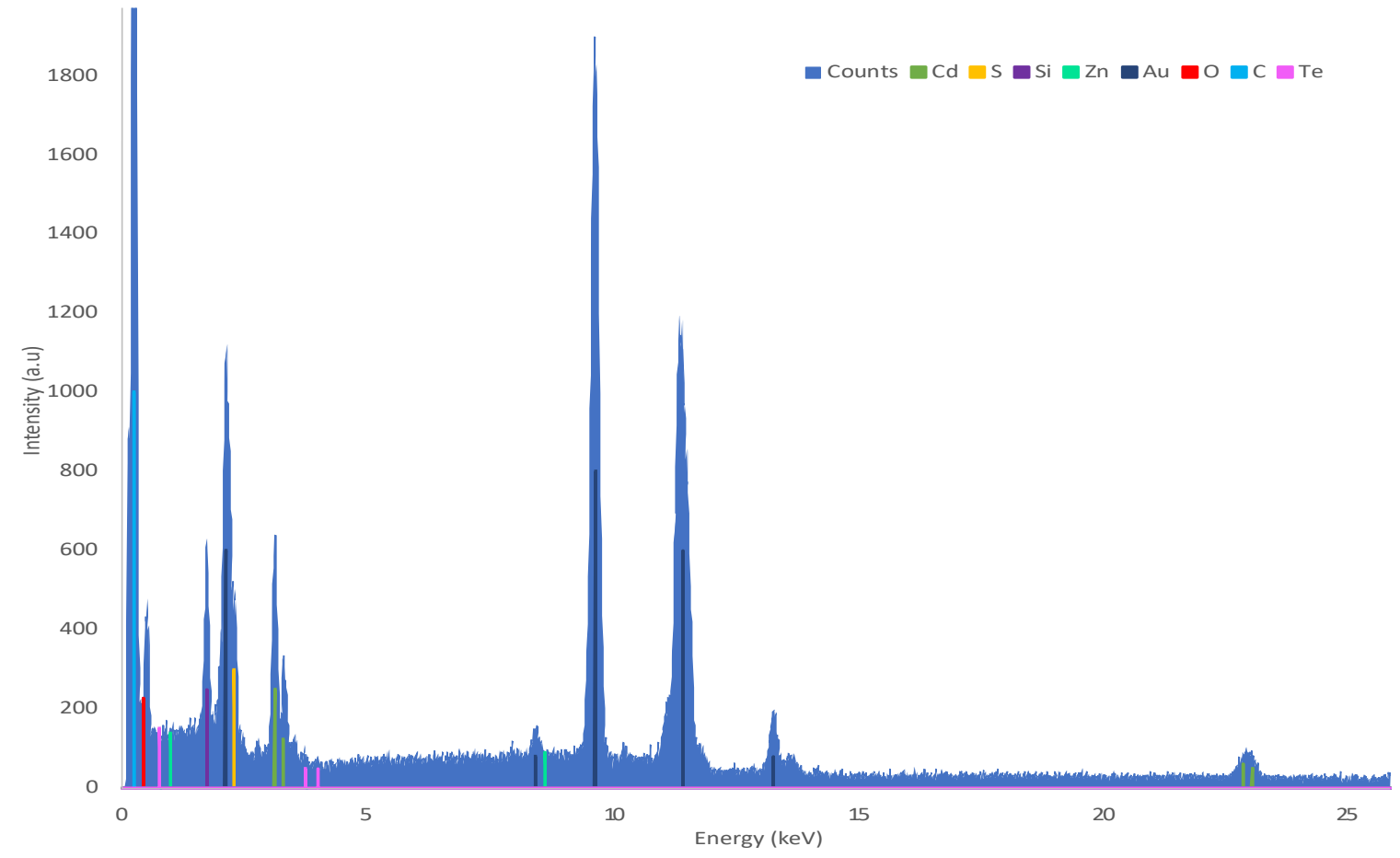

Figure S5. EDX of ZnTe/CdS tetrapods. Strong signals from cadmium and sulfur are observed with weak signals from zinc and tellurium. EDX obtained on gold grids. 

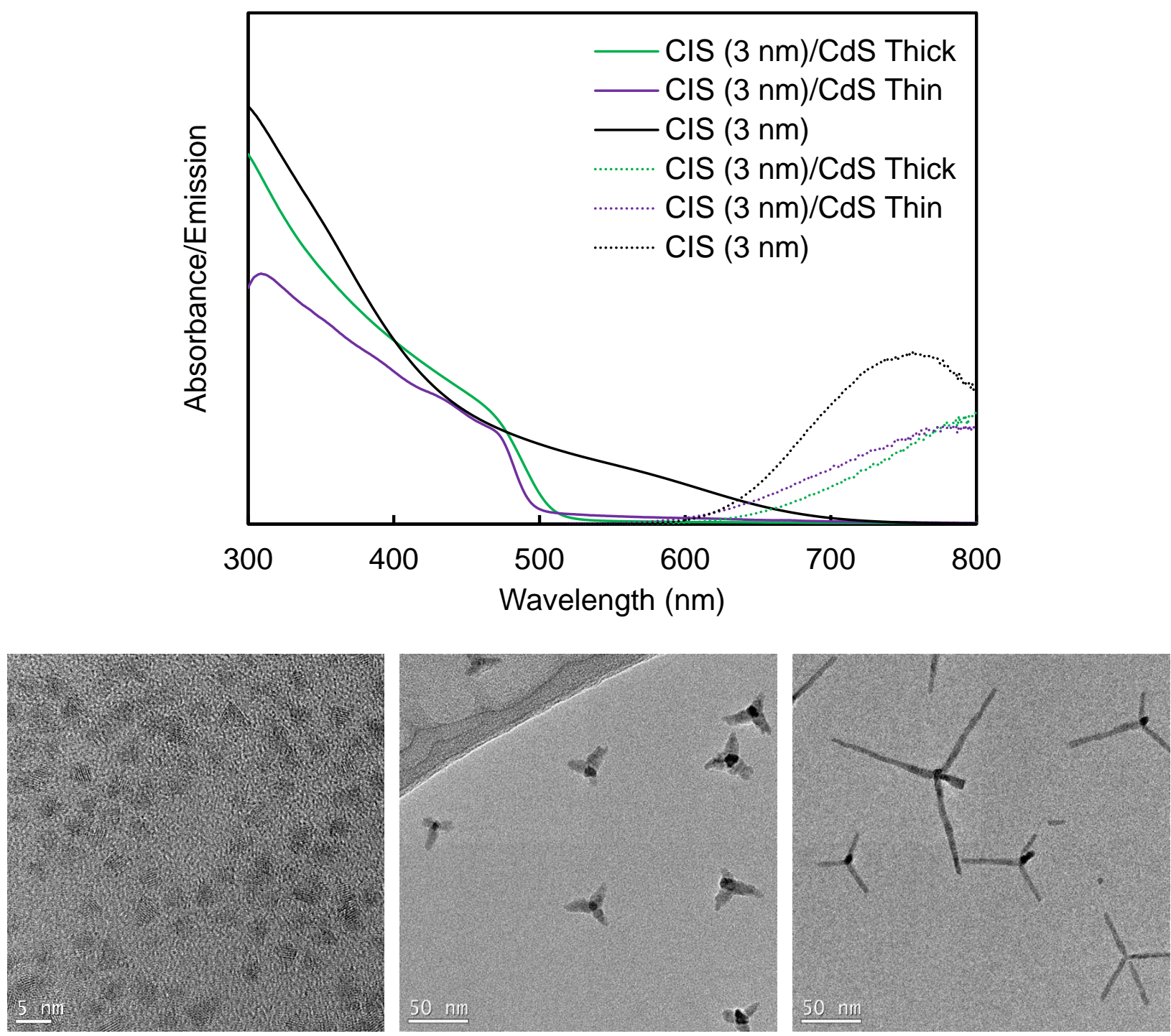

Figure S6. CIS (3 $\mathrm{nm}) / \mathrm{CdS}$ characterization. The absorbance (solid lines) and fluorescence (dotted lines) spectra (top), with TEM images of $3 \mathrm{~nm}$ CIS cores (left), CIS/CdS thick arm tetrapods (middle), and CIS/CdS thin arm tetrapods (right). 

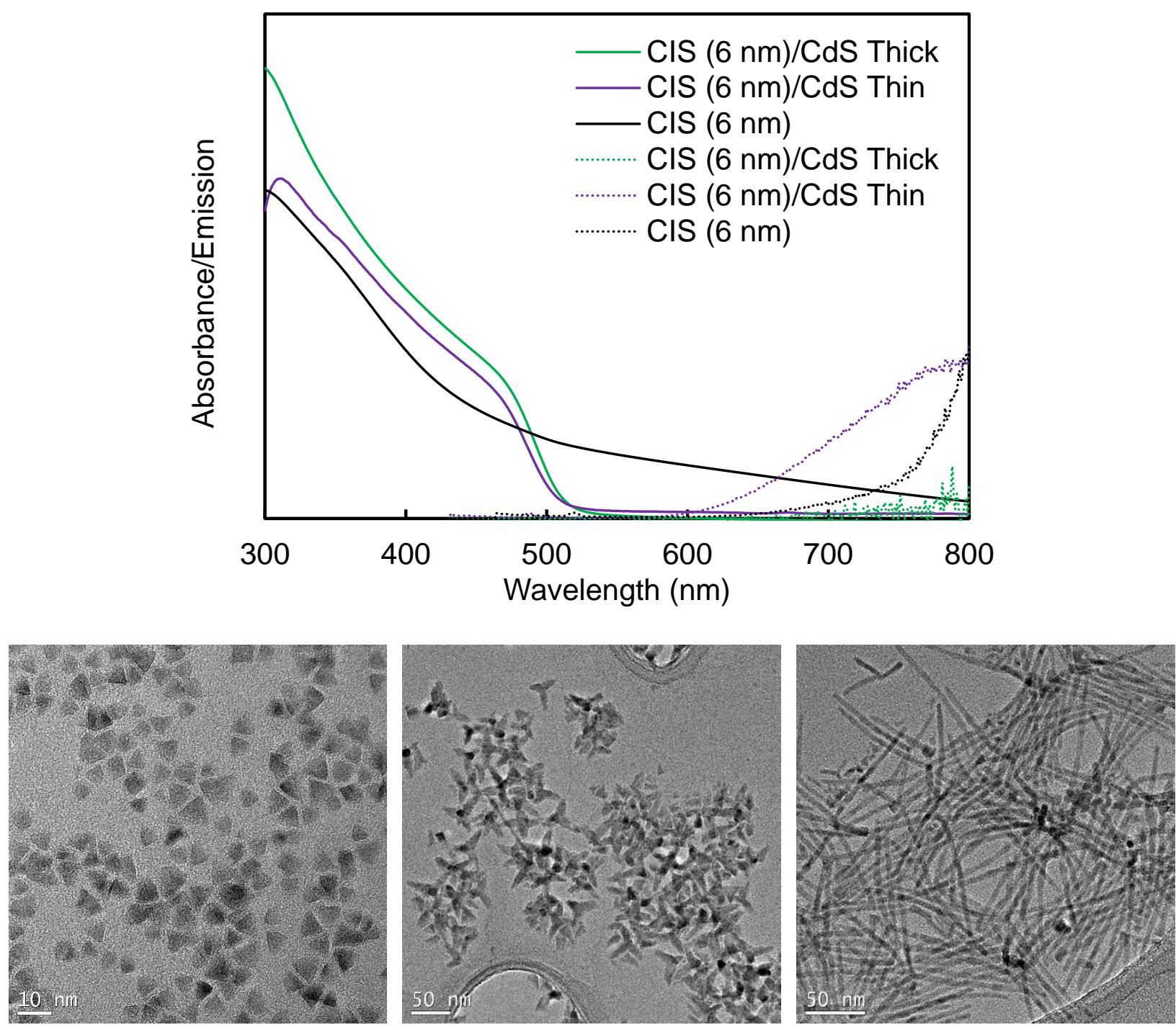

Figure S7. CIS (6 nm)/CdS characterization. The absorbance (solid lines) and fluorescence (dotted lines) spectra (top), with TEM images of $6 \mathrm{~nm}$ CIS cores (left), thick CIS/CdS arm (middle), and thin CIS/CdS (right) arm motifs. 


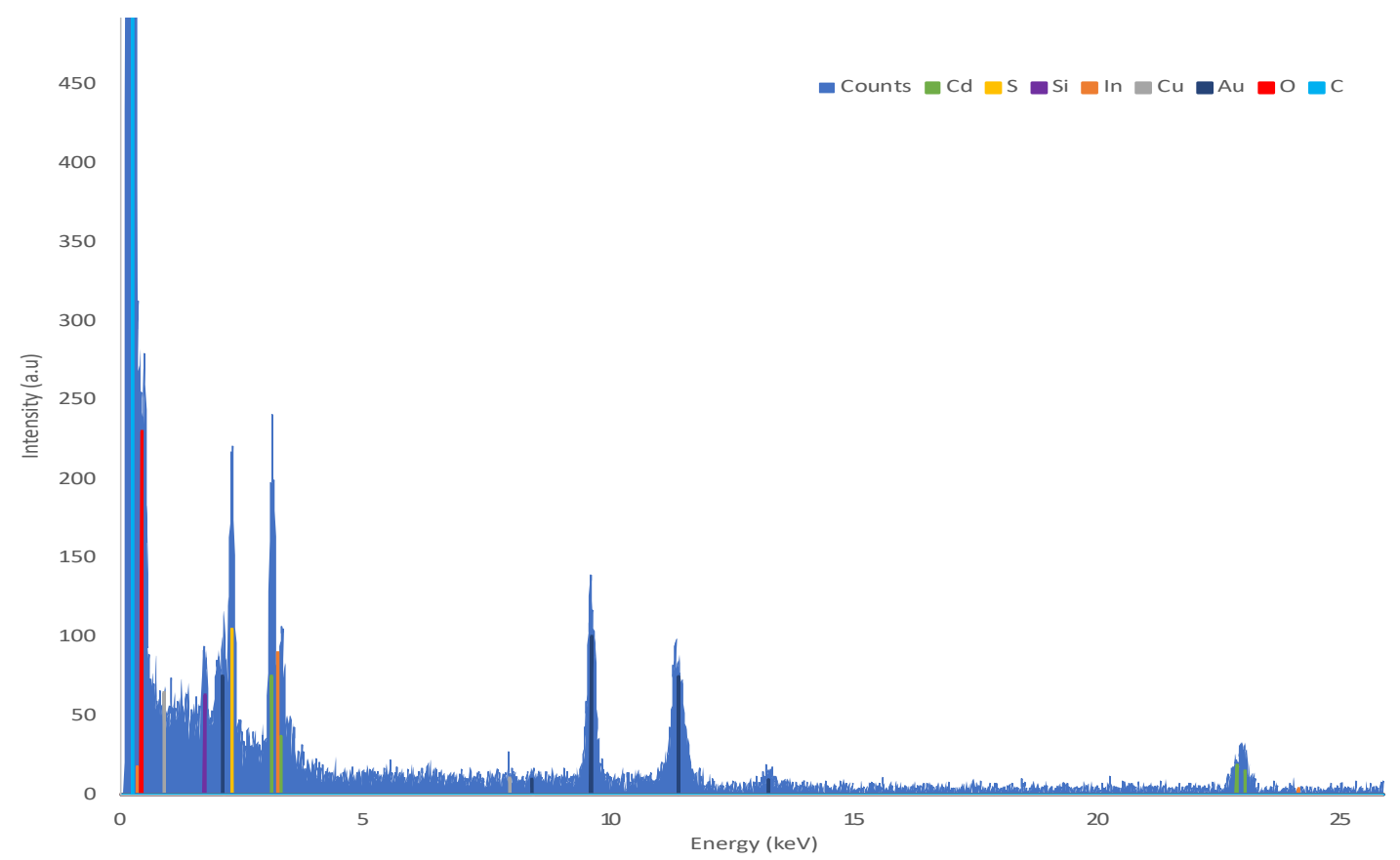

Figure S8. EDX of the core of CIS/CdS tetrapods for $6 \mathrm{~nm}$ CIS seeded tetrapods. EDX obtained on gold grids.

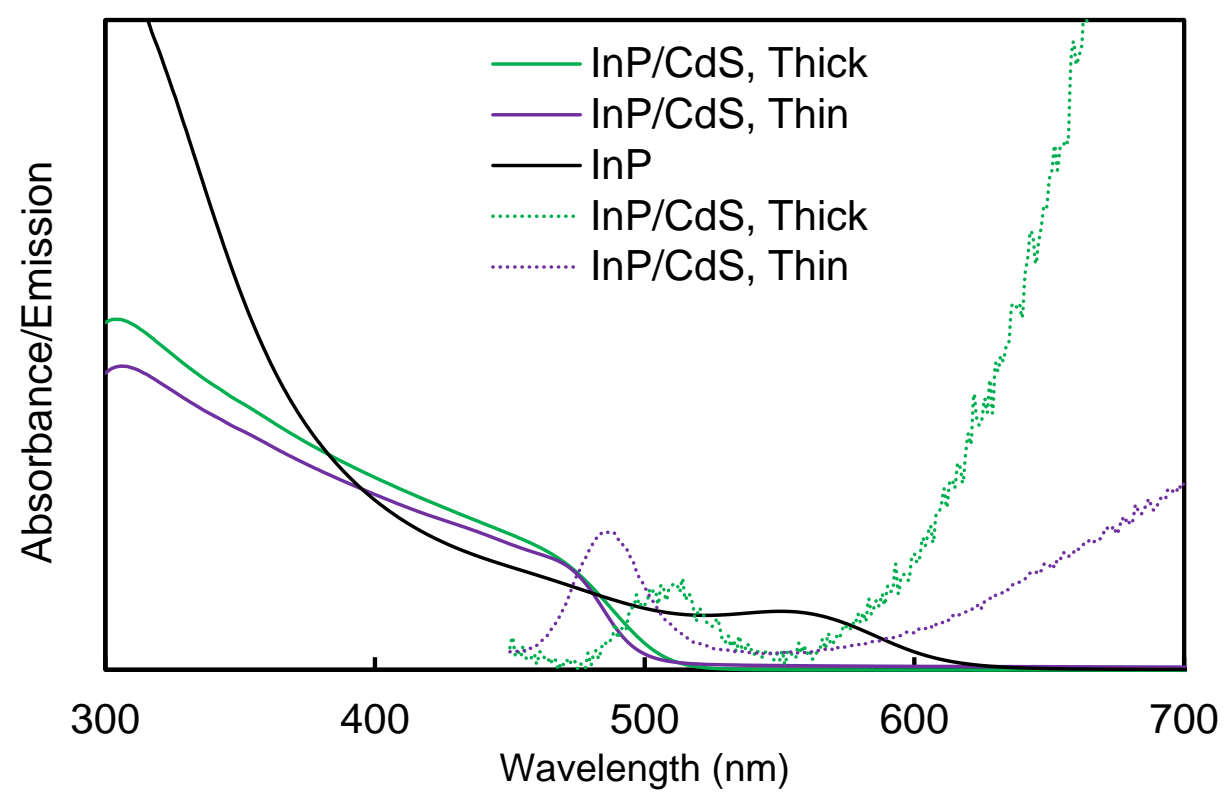

Figure S9. Absorbance (solid lines) and fluorescence (dotted lines) for InP/CdS shows optical properties are primarily dominated by CdS. 


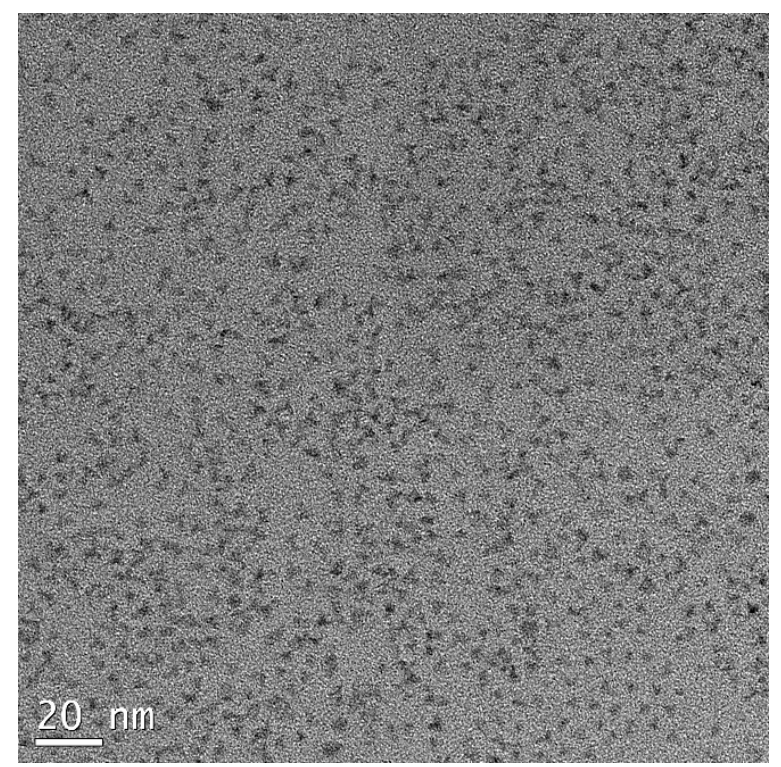

Figure S10. Tetrahedron shaped $3.3 \mathrm{~nm}$ InP cores synthesized from aminophosphine precursor.

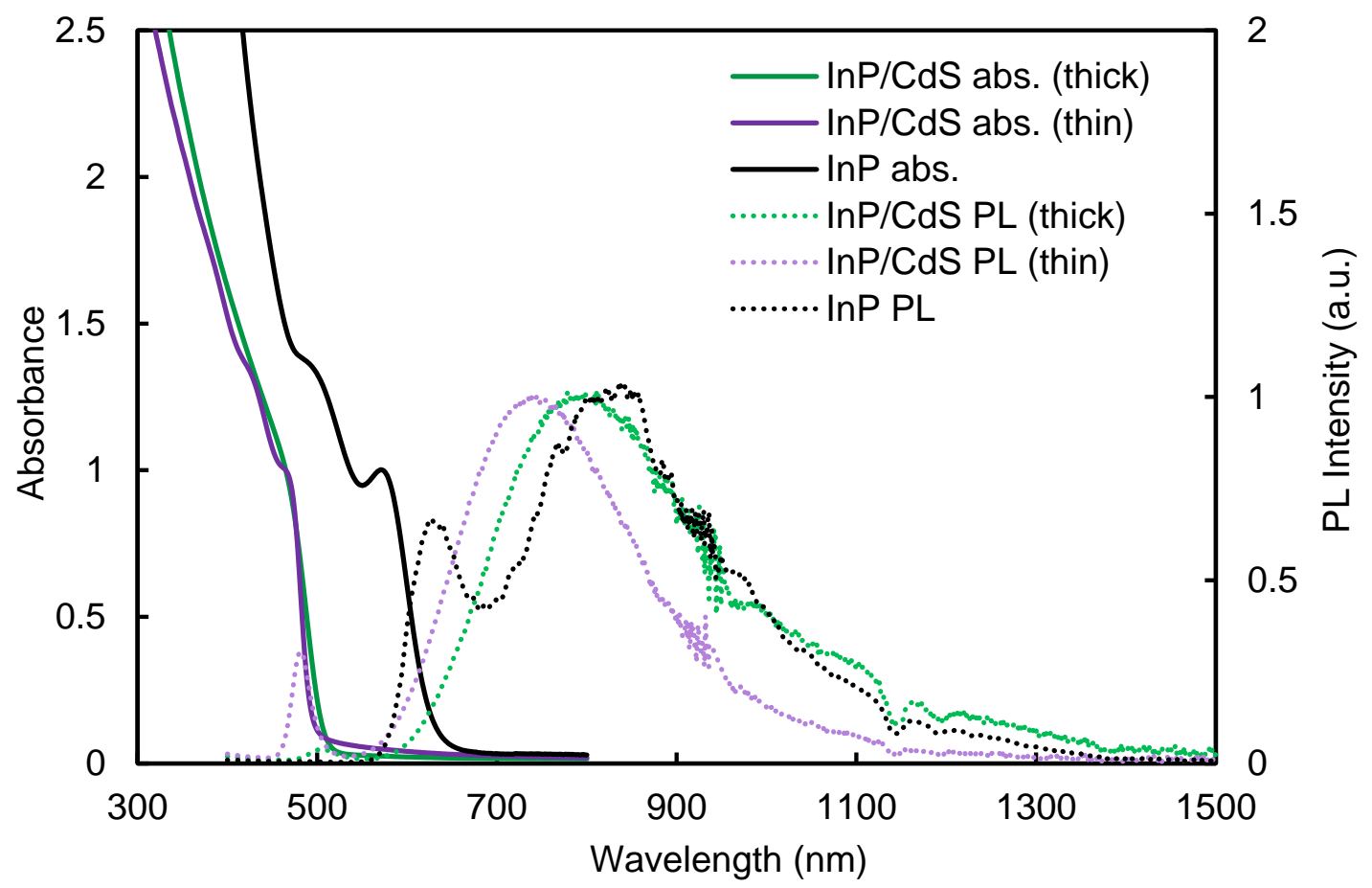

Figure S11. Characterization of InP/CdS from InP tetrahedrons. Absorbance (solid lines) and fluorescence (dotted lines) for InP/CdS (top) along with TEM of InP/CdS in the thick arm (left) and thin arm (right) motifs. 

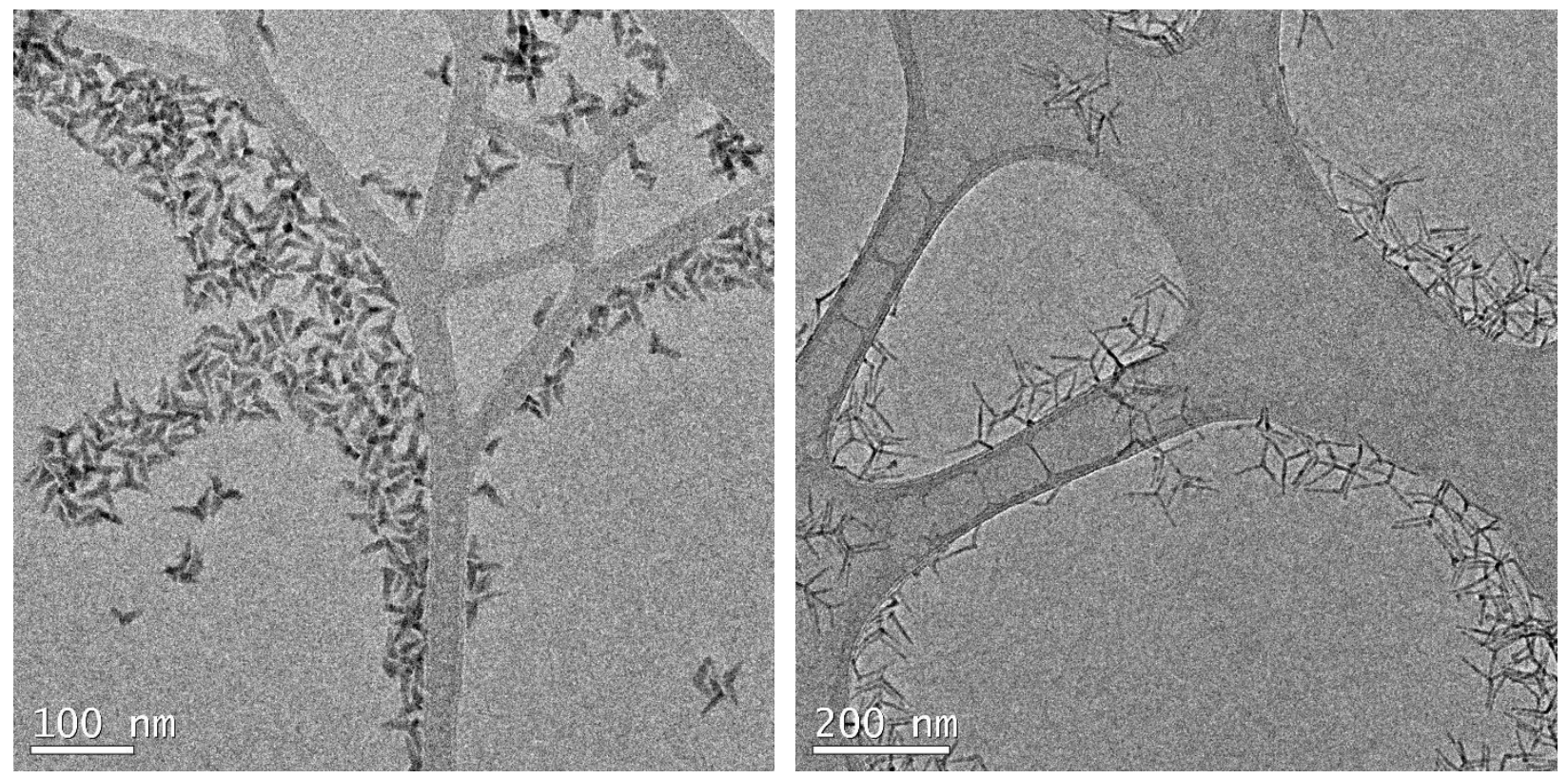

Figure S12. TEM of InP/ZnSeS/CdS tetrapods with thick (left) and thin (right) arms.

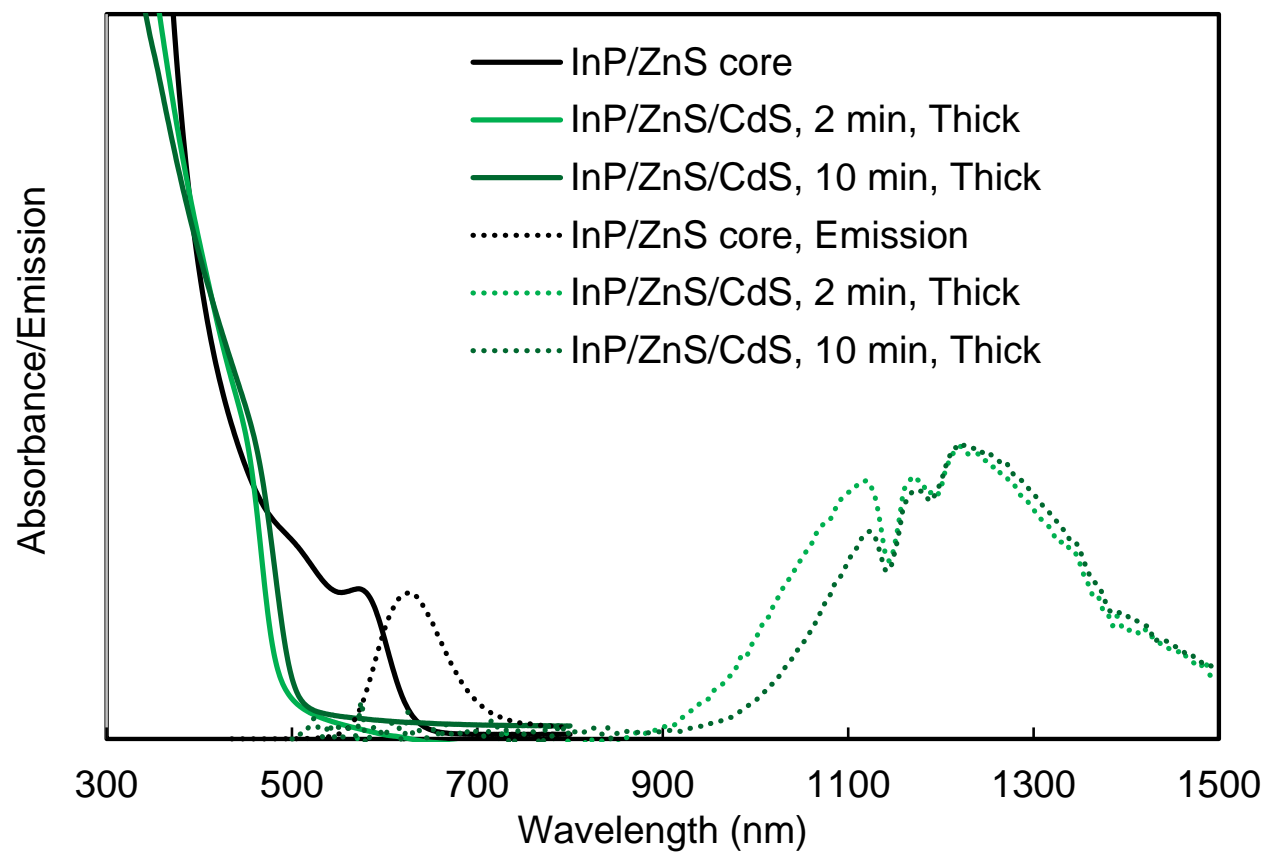

Figure S13. Absorbance (solid lines) and fluorescence (dotted lines) for InP/ZnS/CdS thick arm tetrapod growth over time. 


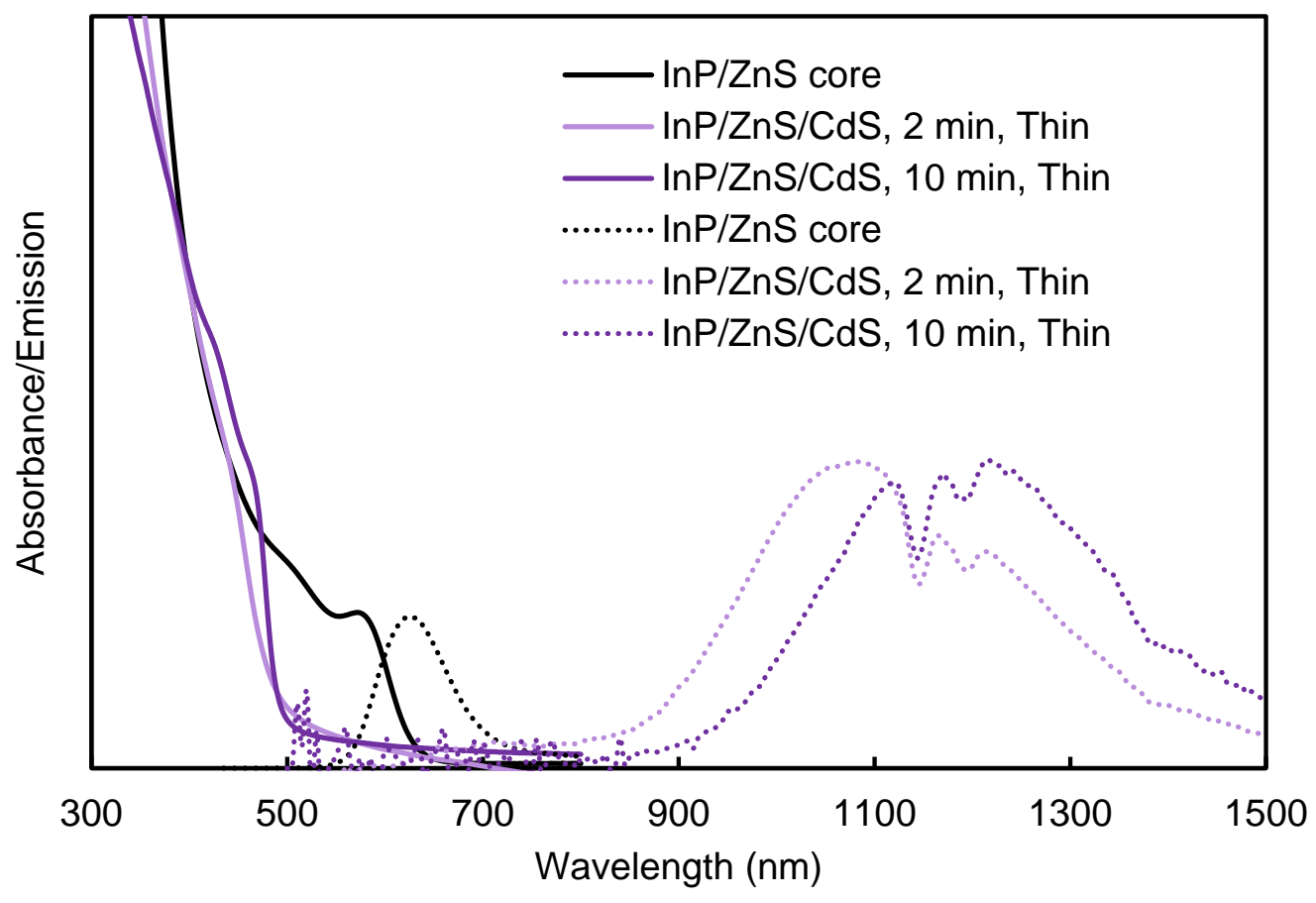

Figure S14. Absorbance (solid lines) and fluorescence (dotted lines) for $\mathrm{InP} / \mathrm{ZnS} / \mathrm{CdS}$ thin arm tetrapod growth over time.
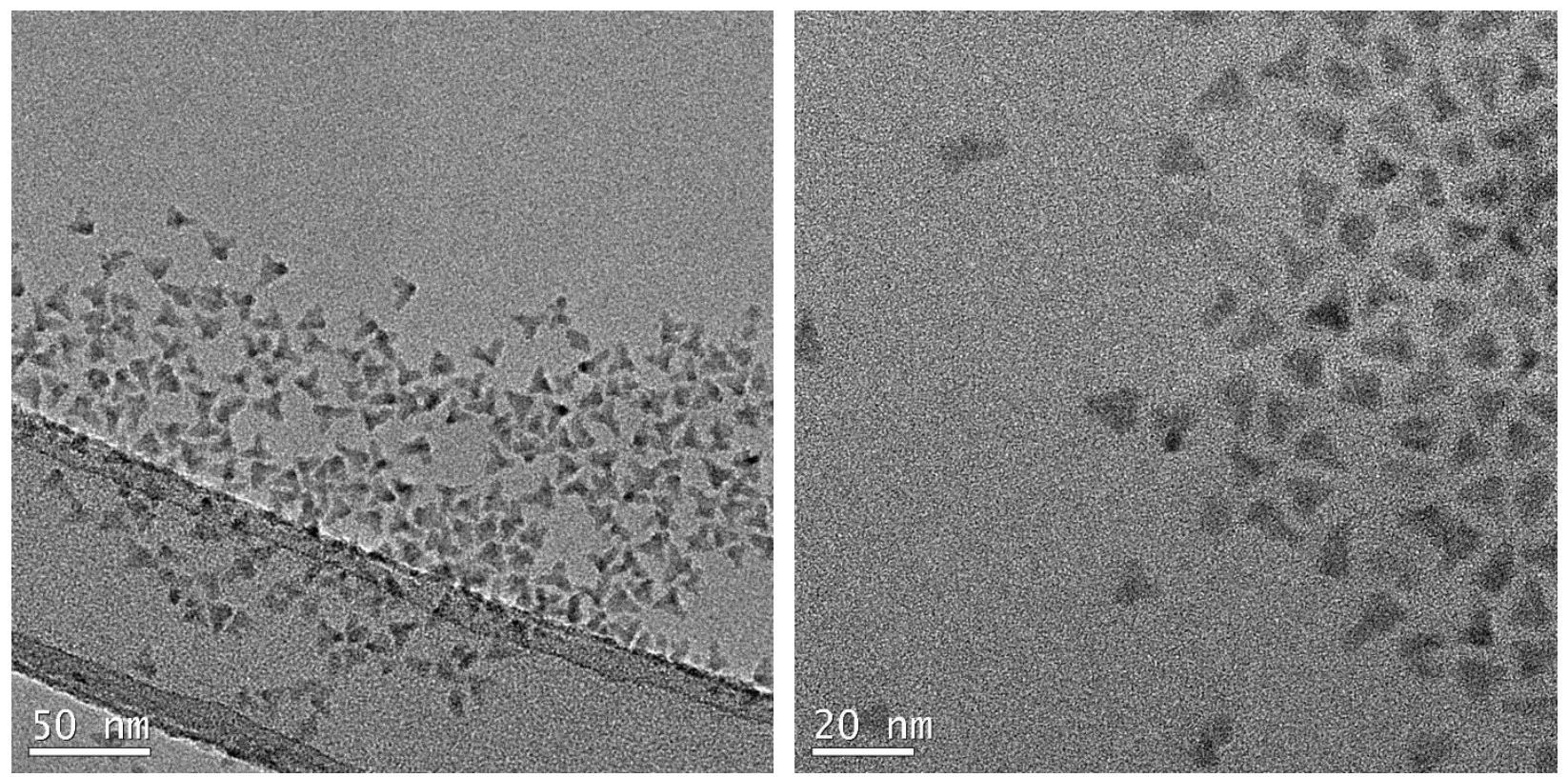

Figure S15. TEM at 2 min for InP/ZnS/CdS (thick on left, thin on right). 


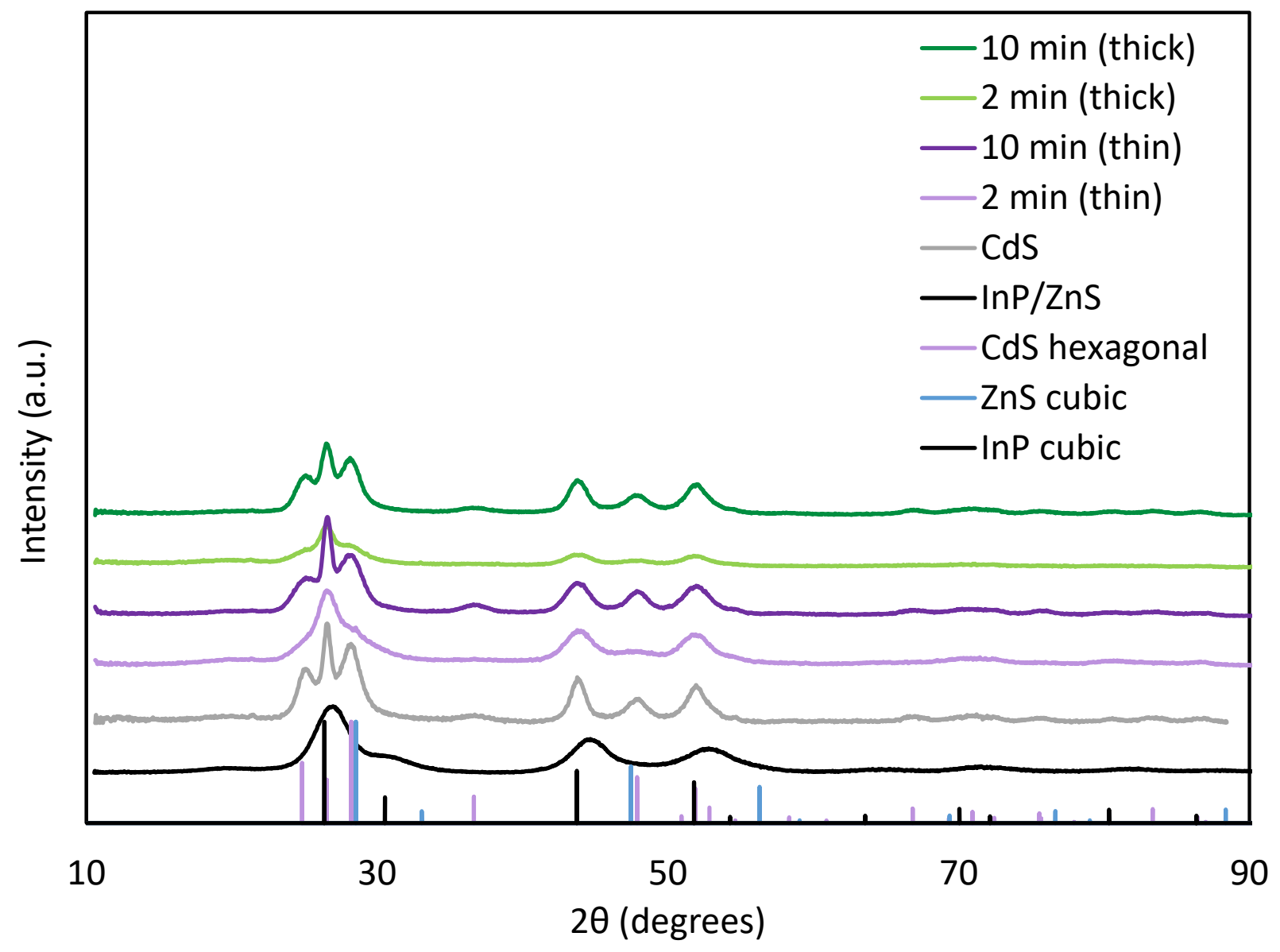

Figure S16. XRD pattern for InP/ZnS seeded tetrapods with CdS arms. Even after 2 min, the signal from the $\mathrm{CdS}$ arms is much stronger than any component from the core. 


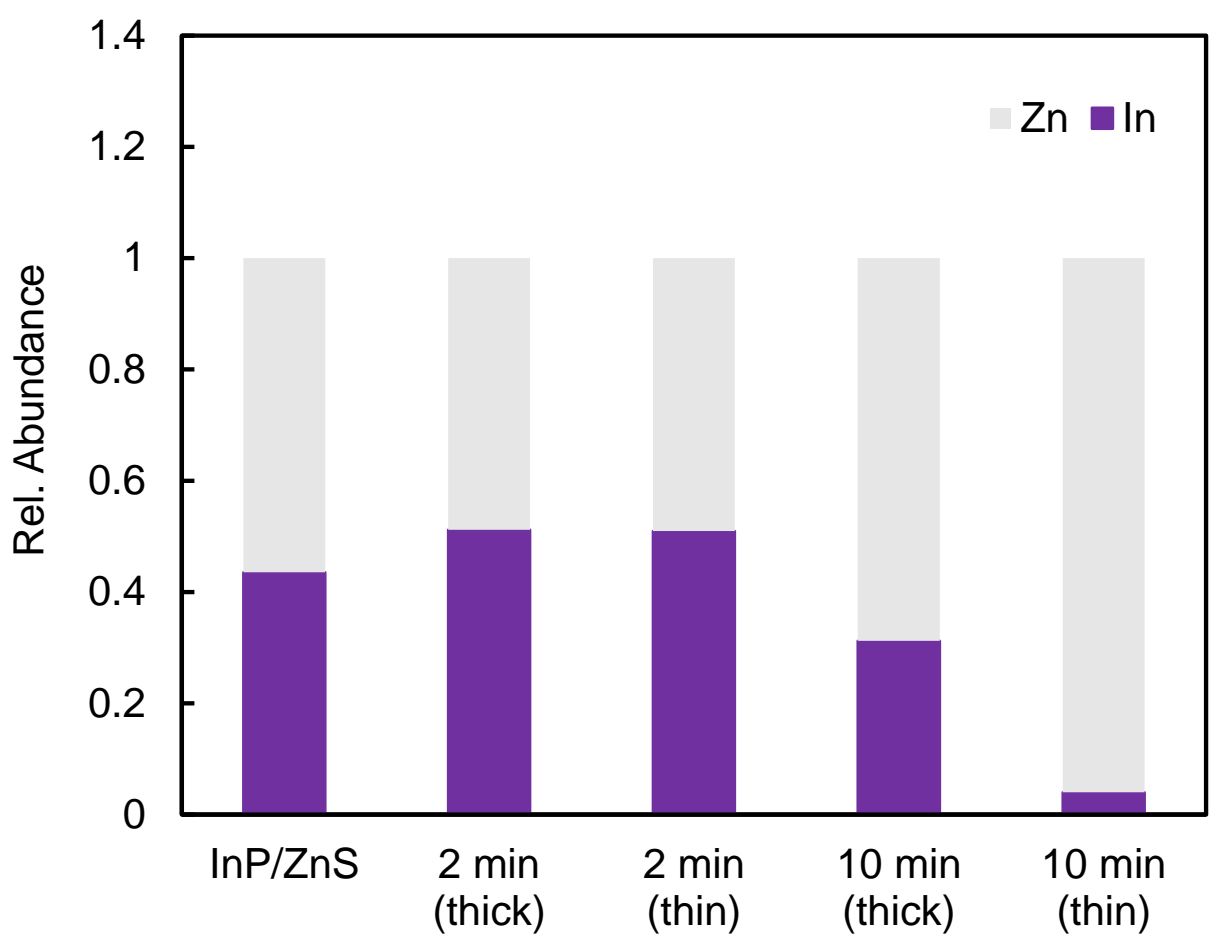

Figure S17. ICP ratios of Indium to Zinc.

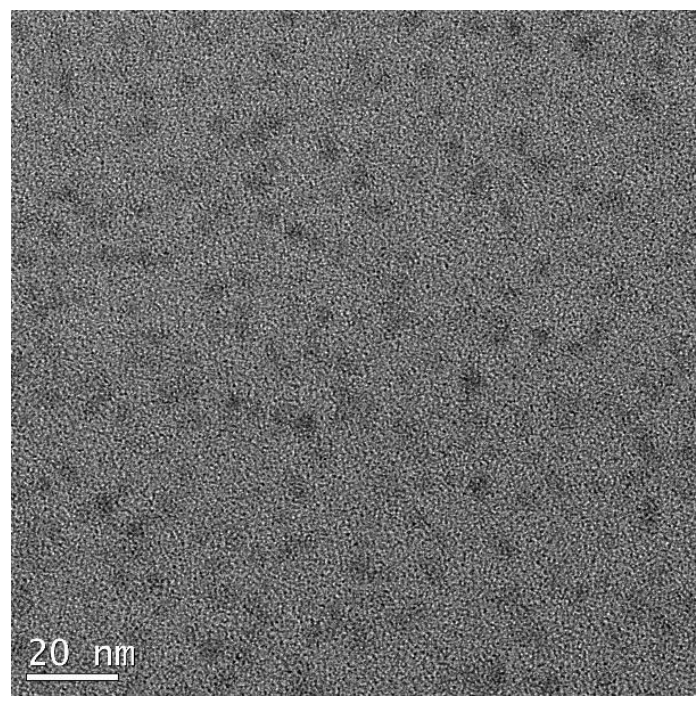

Figure S18. Cores of $3.5 \mathrm{~nm} \mathrm{Cd}_{3} \mathrm{P}_{2}$. 


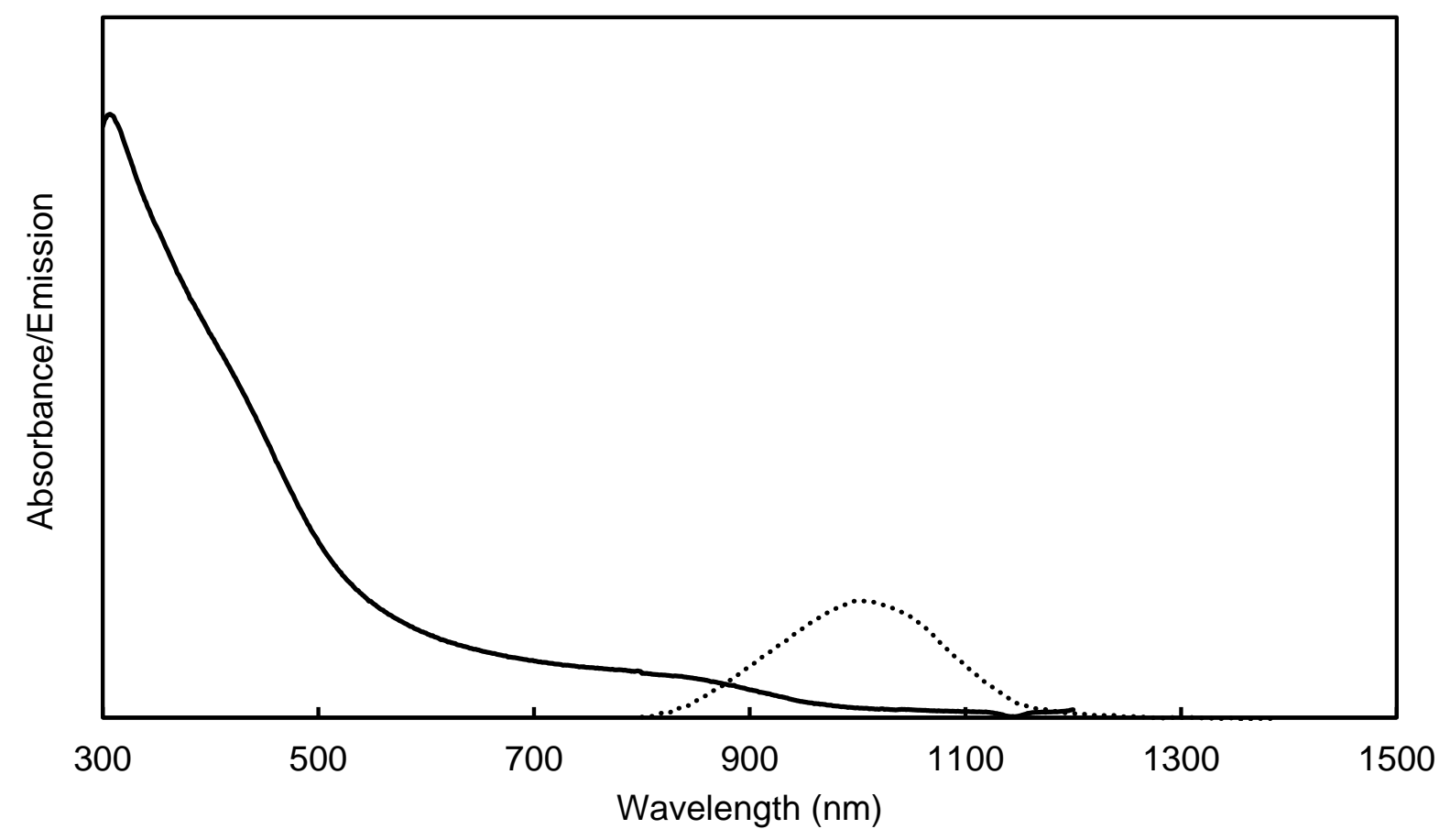

Figure S19. Absorbance (solid line) and fluorescence (dotted line) of $\mathrm{Cd}_{3} \mathrm{P}_{2}$ cores.

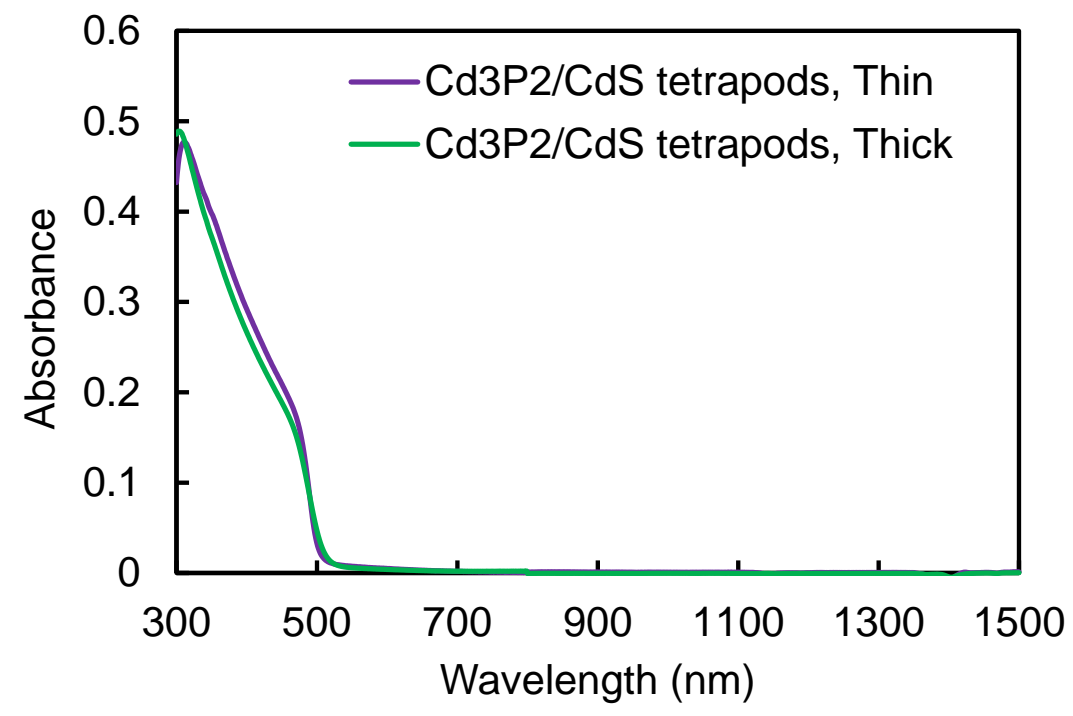

Figure S20. Absorbance of $\mathrm{Cd}_{3} \mathrm{P}_{2} / \mathrm{CdS}$ tetrapods from $3.5 \mathrm{~nm} \mathrm{Cd}_{3} \mathrm{P}_{2}$ cores. 


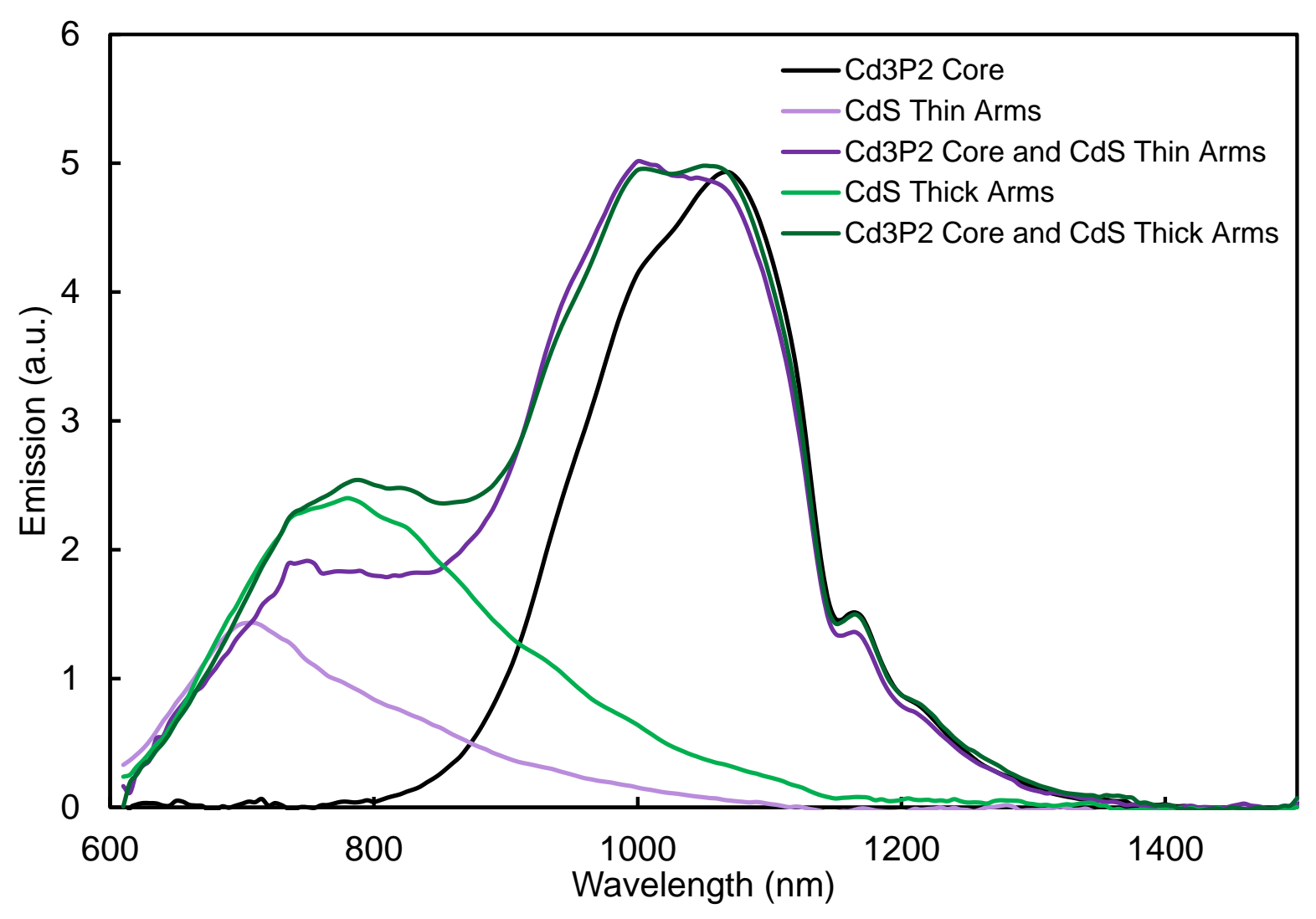

Figure S21. Emission patterns of solutions of $\mathrm{Cd}_{3} \mathrm{P}_{2}$ cores, thin and thick $\mathrm{CdS}$ arms, and mixtures of arms and cores at equal optical densities. 